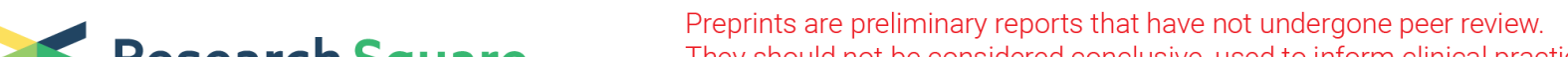 Research Square They should not be considered conclusive, used to inform clinical practice, or referenced by the media as validated information.
}

\section{Extracorporeal Perfusion for Free Fillet Lower Leg Flap After External Hemipelvectomy for Recurrent Osteosarcoma: A New Surgical Technique}

\section{Lorenzo Andreani}

Azienda Ospedaliero Universitaria Pisana

Olimpia Mani ( $\square$ olimpia.mani@gmail.com )

Azienda Ospedaliero Universitaria Pisana https://orcid.org/0000-0003-0276-6096

Edoardo Ipponi

Azienda Ospedaliero Universitaria Pisana

Fabio Cosseddu

Azienda Ospedaliero Universitaria Pisana

Emanuele Cigna

Azienda Ospedaliero Universitaria Pisana

Rodolfo Capanna

Azienda Ospedaliero Universitaria Pisana

\section{Technical note}

Keywords: Extracorporeal Perfusion, Fillet Lower Leg Flap, External Hemipelvectomy, Recurrent Osteosarcoma, Surgical Technique

Posted Date: November 15th, 2021

DOI: https://doi.org/10.21203/rs.3.rs-1044776/v1

License: (c) (i) This work is licensed under a Creative Commons Attribution 4.0 International License. Read Full License 


\section{Abstract}

\section{Background}

External hemipelvectomy often results in exposure of noble structures such as pelvic organs, joint surfaces, iliac neurovascular bundle and bone. Consequently, reconstructive surgery is always challenging. The free fillet lower leg flap is an optimal solution since it allows the transfer of a large amount of tissue and the it determines the absence of donor site morbidities. The prolonged ischemic time represents the weak point of this flap.

\section{Methods}

We presented the case of a 38-year-old man with recurrent radio-induced osteosarcoma arisen on pelvic cavity and involving common iliac vessels, bladder, left ureter and left kidney. We decided to use extracorporeal circulation (ECMO) in order to reduce ischemic time of the flap, since iliac vessels were necessary ligated in the middle of oncologic resection. We perfused the popliteal-based filleted lower leg musculocutaneous free flap with homologue blood and saline solution, while the oncologic dissection was completed.

\section{$\underline{\text { Results }}$}

The free fillet flap remained vital in the immediate postoperative period and in the following months. The patient did not come back to a life-threatening condition at 1y follow-up.

\section{Conclusion}

We believed that ECMO expand the reconstructive indications in those cases in which a complex and long-lasting oncologic resection would make impossible a free flap due to prolonged ischemic time.

\section{Introduction}

Reconstructive surgery after external hemipelvectomy is always challenging. In front of a soft tissue or bone sarcomas, the necessity of wide surgical margins results in exposure of noble structures such as pelvic organs, joint surfaces, iliac neurovascular bundle and bone. The free fillet lower leg flap is a valid reconstructive option when primarily closure firstly and traditional local flap secondly are not sufficient to cover the defect. The main advantages of a lower leg free fillet flap are the lack of donor site morbidities and the possibility to donate large amount of tissue. The prolonged ischemic time represents the weak point of this flap. Some authors have described different methods in order to reduce the ischemic time. However, the ligation of the iliac vascular bundle was always possible at the end of the oncological resection in all the clinical cases presented in literature. Unfortunately, the vessels ligation must be anticipated when tumor involves the internal or external or both iliac arteries. Hence, the need to find alternative strategies that allow the fillet flap survivals. 
We presented the case of a 38-year-old man with recurrent radio-induced osteosarcoma arisen on pelvic cavity and involving common iliac vessels, bladder, left ureter and left kidney.

We decided to use extracorporeal circulation (ECMO) in order to reduce ischemic time of the flap, since iliac vessels were necessary ligated in the middle of oncologic resection. We perfused the popliteal-based filleted lower leg musculocutaneous free flap with homologue blood and saline solution, while the oncologic dissection was completed. This idea was planned by the first and senior authors and it was never described before.

Keywords: free fillet lower leg flap, extracorporeal circulation, bone sarcoma, external hemipelvectomy

\section{Case Presentation}

This report complies with the ethical standards laid down in the 1964 Declaration of Helsinki and its later amendments. The patient gave written consent.

It concerns a 38-year-old man who was diagnosed in May 2000 with Ewing sarcoma affecting his left ilium. The disease was treated with a combination of polychemotherapy and radiant therapy that lasted until March 2001. The patient had complete clinical and radiographical remission and was therefore considered continuously disease free at the following follow ups. In early 2015 he underwent a new diagnostic investigation due to left hip pain and was diagnosed with radiation-induced osteosarcoma of the periacetabular region. Treatment was a combination of systemic chemotherapy and surgical intervention consisting of hemipelvectomy and subsequent reconstruction of the hip bone with a custommade prosthesis.

Between November 2019 and March 2020, the patient was hospitalized due to kidney failure and bilateral hydroureteronephrosis, treated with ureteral stenting. CT scan evidenced an osteogenic mass jutting to the pelvic cavity, responsible for the congestion in the urinary tract. Histological investigations on tissue samples taken from that mass led to diagnosis of locally recurrent osteosarcoma.

In light of the histological nature of the disease, the patient was then treated with chemotherapy and local radiant therapy. This latter, alongside with the neoplastic infiltration, caused an intestinal perforation that was treated with colostomy in an emergency regime. Bowel rupture caused acute septic peritonitis with evidences of intestinal pneumatosis and pneumobilia, treated with combined surgery and antibiotic therapy. Despite surgical intervention, the case developed a colic fistula in communication with the inner surface of the prosthesis. Moreover, through the hospitalization the patient suffered from a severe skin ulceration that finally led to the exposition of the prosthesis in its iliac region. Lack of skin coverage, in combination with the anatomical communication with the intestine caused a complex infection of the prosthesis.

When the patient arrived to our attention the skin was heavily macerated and hot to the touch in the trochanteric, gluteal and iliac region. In this latter a large and deep ulceration exposed the custom-made 
prosthesis to the external agents (FIG.1). Left hip range of movement was severely reduced and both active and passive mobilization caused severe pain. We urgently subjected him to MRI and CT-scan, in order to gain a better understanding of the local anatomy, evaluate the tissues and organs that could have been damaged or involved by the neoplasm, but also plan the most suitable surgical approach.

CT images were partially limited by the metal artifacts caused by the custom-made prosthesis.

Common iliac artery was patent from its origin for $3.5 \mathrm{~cm}$, whereas its distal portion, as well as its terminal branches were englobed by the osteosarcoma. Distally, femoral artery was not channeled at its root. Left kidney and urinary tract suffered from severe ureteronephrosis. Kidney iodine intake was completely abolished and the ureter was surrounded by neoplastic tissue. Right upper urinary apparatus, for its part, maintained subnormal morphology and functionality. Neoplastic tissue appeared to involve the lateral left surface of the urinary bladder, without clear involvement of the vesical trigon.

MRI images defined the recurrence of osteosarcoma was $14 \mathrm{~cm}$ long and about $10 \mathrm{~cm}$ wide, located anteriorly to rectum, sigma and sacrum. They confirmed he mass infiltrated both the internal and external iliac vessels on the left, while on the right it appeared contiguous to the iliac bifurcation without evidences of neoplastic involvement. Vesical trigon appeared free of disease as well. MRI also showed diffused signal alterations nearby the custom-made prosthesis.

Due to the complex clinical picture and the multiorgan injury, we planned and performed the intervention on a multidisciplinary basis taking advantage of the collaboration with our institution's teams of orthopedics, anesthesiology, general surgery and plastic surgery.

\section{SURGICAL INTERVENTION}

In the operative room the patient received general anesthesia and was set on a radiolucent surgical table in a supine position. The stoma and the area of ulceration with prosthesis exposition were both isolated and protected through surgery.

General surgeons performed longitudinal median laparotomy on the pre-existing wound, approach complicated by the several adhesions that resulted from the previous interventions. Through the procedure surgeons palped a thick nodule within the abdominal wall; the mass was eradicated and intraoperative histological analysis confirmed its malignant nature. Abdominal cavity was then explored, the remnant colon was mobilized and the inferior cave vein and aorta were exposed until their bifurcation. Left kidney and the urinary tract were clearly involved by idroureteronephrosis and the left ureter, in particular, was secreting purulent material and seemed dislocated by the neoplastic mass. The urinary bladder appeared slightly increased in volume, thick and hypoelastic as a result of the radiant therapy. Its lateral and posterior wall were hardly clivable from the nearby tumor. The vesical trigon, instead, appeared free of disease and a narrow space could be evidenced between it and the neoplastic mass on its left. The team therefore sacrificed the part of the organ involved by the disease performing a left 
hemicystectomy. Resection was also spread to the upper urinary tract and kidney, which were also removed, and to the left genital system, since left testicle was removed by retrograde orchiectomy.

In a second phase our orthopedic team extended the incision to the left inguinal region to surround the superior pubic ramus and expose the inferior one which were resected sequentially. Afterwards, adductor muscles were dissected and in parallel the obturator nerve and vessels were sacrificed. The patient was then slightly rotated on his right side to reach the semi-lateral position and allow a better view also of the gluteal and sacral region. Section of the left gluteal musculature and spino-pelvic muscles was allowed and therefore performed. The patient was subsequently set back to a supine position and a pneumatic tourniquet was placed and activated at the root of the left lower limb.

We therefore proceeded to harvest the free miocutaneous flap from the left leg of the patient (Fig. 2). Triceps surae was isolated from the surrounding soft and bone tissues and the popliteal vascular bundle was identified, ligated and dissected at its origin (Fig. 3). Harvesting, as a whole, lasted almost 90 minutes.

The flap, now completely free, was set aside on a separate surgical table and reperfused using the ECMO (ExtraCorporeal Membrane Oxygenation) system that maintained tissue vitality by circulating 2 units of blood red cells and saline solution. Popliteal artery and vein were canulated (FIG.4). The flap laid on sterile gauzes soaked in saline solution in order to keep hydrate the exposed muscles.

In parallel, on the main surgical field, the demolitive phase was brought to an end. Left common iliac vessels were clamped and resected, left nerve roots arising from lumbar segments were severed, the remaining urinary bladder was sutured and finally a longitudinal osteotomy was performed to divide the left sacral wing from the rest of the bone. Once the resection was over, the left pelvic arch - from the sacral wing to the pubic rami - could be removed in block alongside with the rest of the lower limb. The tissue deficit in the left pelvic region was finally fulfilled with the free miocutaneous flap whose vitality had been successfully maintained by the extracorporeal circulation device. Termino-terminal anastomoses, both arterious and venous, were carried out between left common iliac vessels and flap's vascular bundle. Once reperfused the flap appeared vital and healthy. Two surgical drainages were placed under the flap, which was sutured to the nearby tissues.

\section{POST-OPERATIVE CONDITIONS}

Once surgery was over, the patient was subjected to peri-operative monitoring in regimen of intensive care for a couple of days. General conditions were stabilized and the clinical conditions remained stable for the first days, with a subsequent slow but progressive improvement of the overall picture as the weeks passed. In fact, although hospitalization lasted for more than a month after the intervention, the patient did not come back to a life-threatening condition (1 year FU). The myocutaneous flap did not show signs of necrosis and remained healthy throughout the post-operative phase, successfully taking roots in the receiving site. 


\section{Discussion}

External hemipelvectomy for treatment of pelvic sarcomas resulted in a great loss of substance, which determined the exposure of pelvic organs such as bladder and rectum, the iliac neurovascular bundle, the hemisacrum and peritoneal contents. Reconstruction is challenging and patient tailor-made. Several flaps were described to cover external hemipelvectomies.

The gluteus maximus musculocutaneous flap, usually utilized for the treatment of decubitus, was well described by Becker ${ }^{1}$ and Dirnberger ${ }^{2}$ and many other authors. It is vascularized from the inferior gluteal artery and many variations of this flap were possible. In our case, it was excluded because left gluteal arteries were compromised from previous radiotherapy and gluteus muscle was invaded by the tumor. Anyway, even if gluteal musculature was healthy, it would not be enough to cover the expected loss of substance.

The rectus abdominus musculocutaneous flap was anatomically described in 1988 by Moon and Taylor ${ }^{3}$. It is a well- vascularized versatile pedicled flap based on the deep inferior epigastric vessels and it is able to cover large pelvic loss of substance. The ipsilateral flap could be associated to high complication rate due to dissection, which took place in the immediate vicinity of the deep inferior epigastric pedicle. Therefore, it could be safer using a flap based on the contralateral pedicle 4 . Unfortunately, in our patient abdominal wall was contaminated by the tumor; thus, it would not be an oncological safe reconstructive choice. Moreover, the patient was colostomized before the surgery.

The anterior myocutaneous flap of quadriceps femoris muscle ${ }^{5}$, generally indicated for buttock tumors, was excluded because the malignancy extended anteriorly in the pelvic cavity with involvement of the external and internal iliac vessels. As a matter of fact, we used the common iliac vessels to perform the anastomosis. For the same reason, a discontinuous myocutaneous quadriceps flap supplied by the superficial femoral artery ${ }^{6}$ was not indicated.

Although more favorable due to the absence of ischemic time and for shorter operating time, a pedicled flap were not suitable for our patient. Therefore, we decided to perform a free tissue transfer.

The type of surgery we offered the patient was a palliative surgery, strongly desired by himself and his family in order to make the last period of life free from pain, bleeding, odor and facilitating nursing care. Therefore, the lack of donor site morbidities and rapid recovery from surgery was crucial in front of a surgical palliation.

Latissimus dorsi free flap represented the main solution to reconstruct large loss of substance after hemipelvectomy. However, it would have resulted in shoulder girdle instability, compromising patient's balance and his residual quality of life.

Once all traditional possibilities have been explored, the spare parts surgery was the only one which fulfilled oncological and palliative goals. 
"Spare parts" concept involved the use of composite tissues from the amputated limb to reconstruct complex defects originated from tumors or trauma. ${ }^{7}$

The Popliteal- based filleted lower leg musculocutaneous free-flap was described for the first time by Workman et al. in $1991^{8}$. A free fillet flap from the amputated limb maintained the possibility of LD free flap as second chance in case of failure.

One of the principal problems of this flap was the prolonged ischemic time. Few solutions were described in medical literature, due to the small number of patients who needed this kind of surgery.

Yamamoto et al. ${ }^{9,10}$ harvested the flap after the extensive resection of the tumor, just before ligating the vessels and removing the limb and they immediately cooled the fillet flap with crushed ice. Thus, the time of ischemia of the flap, which was minimized from icing the flap, coincided with the iliac vessels preparation for anastomosis.

In our experience, the clinical condition and the extension of malignancy have resulted in the need for a simultaneous approach between orthopedics, general surgeons and plastic surgeons. In particular, tumor involved bladder, urinary tract including left kidney and enveloped the iliac external and internal vessels. Therefore, it was not possible to ligate the iliac vessels at the end of the oncological resection. Once the vessels were ligated, the abdominal surgery had to be completed and this would have resulted in a time of flap's ischemia not compatible with its survival, even if cooled with ice.

Bibbo et al. ${ }^{11}$ suggested to start the surgery with the fillet flap dissection, leaving the flap attached to the proximal popliteal vascular pedicle and the Achilles tendon. In this way, they allowed the fillet flap perfusion during oncologic resection. They reported an ischemic time less than 45 minutes, time needed for preparation of vascular pedicle.

This method was not usable in our case too; either for the need of early ligation of iliac vessels, or for the necessity to frequently rotate the patient on the operating table in order to reach the malignancy in every parts. This would have entailed dangerous stresses on the vascular pedicle, risking to create damage. Moreover, our patient had zero negative blood type. The flap perfusion during dissection would have increased blood loss, enhancing the demand of transfusion for a blood type not available in large quantities.

Finally, Boehmler et al. ${ }^{12}$ described a two-stage fillet flap in order to decrease ischemic time and to allow patient resuscitation between the two stage. We preferred to avoid two surgeries, given the clinical frailty of our patient and the need for complex and simultaneously cooperation of three different teams.

Furthermore, some authors preferred to harvest the flap preserving the fibula in order to save time and to not risk to damage the flap vascular bundle ${ }^{12-14}$. Contrary, we decided to make a subperiosteal dissection, removing completely the fibula. In our opinion, leaving the fibula inside the flap could determine a risk of decubitus that is higher than the potential benefits. 
In light of all these issues, the first and senior authors devised a new strategy never described before. As used in transplant surgery, we decided to connect the flap to the ECMO system by circulating 2 units of blood red cells and saline solution. This strategy allowed to save the time it took to finish abdominal oncological resection after vessels ligation and lastly the osteotomy of the sacrum. The difficulty encountered by the hemodynamics' technician was that the free flap consisted of an open circuit due to the dispersion of terminal collateral arteries. Consequently, pump pressure set on the machine was hardly supported. The problem was solved infusing saline solution continuously into the circuit and making adequate hemostasis simultaneously on the periphery of the flap. Moreover, the flap was closed in on itself in order to reduce loss of circulating volume.

The perfusion lasted above 60 minutes and was successful. At the end the flap was rosy and vital with a good refill time.

\section{Conclusion}

Free fillet lower leg flap is useful for large loss of substance, when traditional flaps are not sufficient to cover the defect. The donor site morbidity is absent. In effect, it allows to avoid free flaps such as the LD, which could create a shoulder instability and could compromise the balance of the patient, already unbalanced from the monopodalic support resulting from amputation.

The usage of this flap could be hindered by the ischemic time that will be the longer the earlier it is necessary to interrupt iliac vessels during oncologic resection.

In conclusion, we believed that ECMO expand the reconstructive indications in those cases in which a complex and long-lasting oncologic resection would make impossible a free flap due to prolonged ischemic time.

\section{Declarations}

\section{Ethics approval:}

not applicable

\section{Consent for publication:}

the patient gave written consent

\section{Availability of data and materials:}

data sharing is not applicable to this article as no datasets were generated or analysed during the current study 


\section{Competing interests:}

the authors declare that they have no competing interests

\section{Funding:}

none

\section{Authors' contributions:}

all the authors contributed equally

\section{Acknowledgements:}

not applicable

\section{References}

1. Becker, H. The distally-based gluteus maximus muscle flap. Plast. Reconstr. Surg. 63, 653-656

2. Dirnberger, F. The nontypical gluteus maximus flap. Plast. Reconstr. Surg. 81, 567-576 (1988).

3. HK, M. \& GI, T. The vascular anatomy of rectus abdominis musculocutaneous flaps based on the deep superior epigastric system. Plast. Reconstr. Surg. 82, 815-832 (1988).

4. Ross, D. A. et al. Soft tissue reconstruction following hemipelvectomy. Am. J. Surg. 176, 25-29 (1998).

5. Sugarbaker, P. H. \& Chretien, P. A. Hemipelvectomy for Buttock Tumors Utilizing an Anterior Myocutaneous Flap of Quadriceps Femoris Muscle.

6. Lotze, M. T. \& H. Sugarbaker, P. Femoral artery based myocutaneous flap for hemipelvectomy closure: Amputation after failed limb-sparing surgery and radiotherapy. Am. J. Surg. 150, 625-629 (1985).

7. MV, K. et al. The concept of fillet flaps: classification, indications, and analysis of their clinical value. Plast. Reconstr. Surg. 108, 885-896 (2001).

8. ML, W., DF, B. \& BL, C. Popliteal-based filleted lower leg musculocutaneous free-flap coverage of a hemipelvectomy defect. Plast. Reconstr. Surg. 89, 326-329 (1992).

9. Yamamoto, Yuhei; Minakawa, Hidehiko; Takeda, N. Pelvic reconstruction with a free fillet lower leg flap. Plast. Reconstr. Surg. 99, 1439-1441

10. Yamamoto, Y. \& Sugihara, T. Pelvic reconstruction with a free fillet lower leg flap. Plast. Reconstr. Surg. 111, 1475-1476 (2003). 
11. Bibbo, C., Newman, A. S., Lackman, R. D., Levin, L. S. \& Kovach, S. J. A simplified approach to reconstruction of hemipelvectomy defects with lower extremity free fillet flaps to minimize ischemia time. J. Plast. Reconstr. Aesthetic Surg. 68, 1750-1754 (2015).

12. Boehmler, J. H., Francis, S. H., Grawe, R. K. \& Mayerson, J. L. Reconstruction of an External Hemipelvectomy Defect with a Two-stage Fillet of Leg-Free Flap. J. Reconstr. Microsurg. 26, 271276 (2010).

13. KJ, T. \& EB, T. Free filet leg flap. Clin. Orthop. Relat. Res. 182-185 (2001). doi:10.1097/00003086200104000-00028

14. Roulet, S. et al. Free fillet lower leg flap for coverage after hemipelvectomy or hip disarticulation. Orthop. Traumatol. Surg. Res. 105, 47-54 (2019).

\section{Figures}

\section{Figure 1}

Patient in lateral decubitus. The image showed the deep ulceration exposed the infected custom-made prosthesis to the external agents.

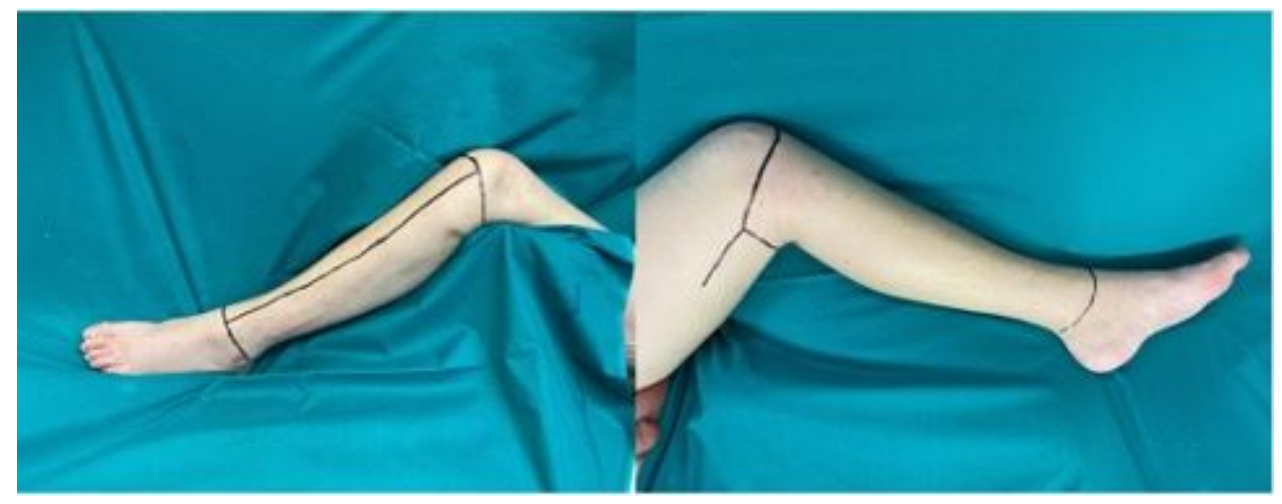

\section{Figure 2}

The triceps surae free fillet flap design 


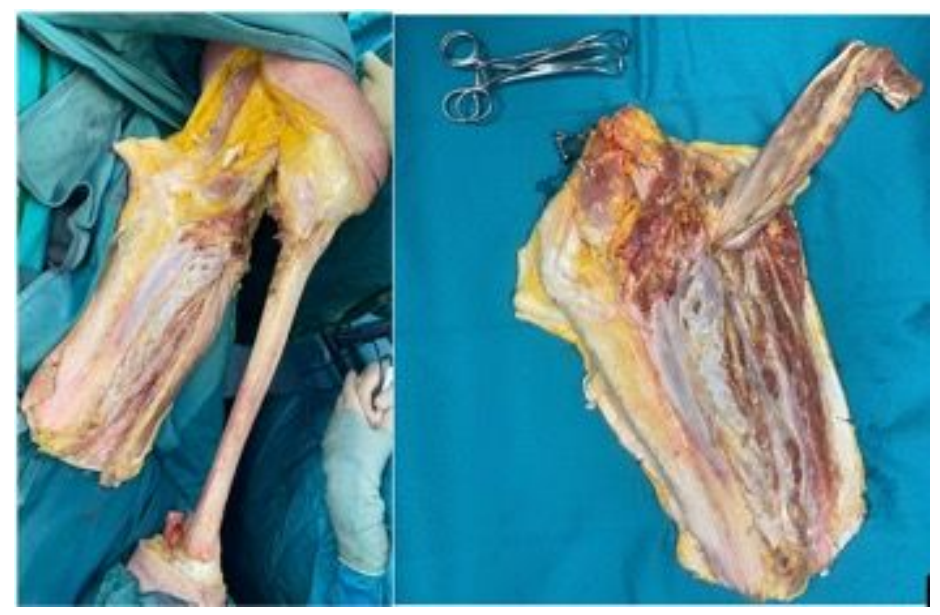

\section{Figure 3}

The free miocutaneous flap harvested from the left leg of the patient. On the right the popliteal vascular bundle was identified, ligated and dissected at its origin.

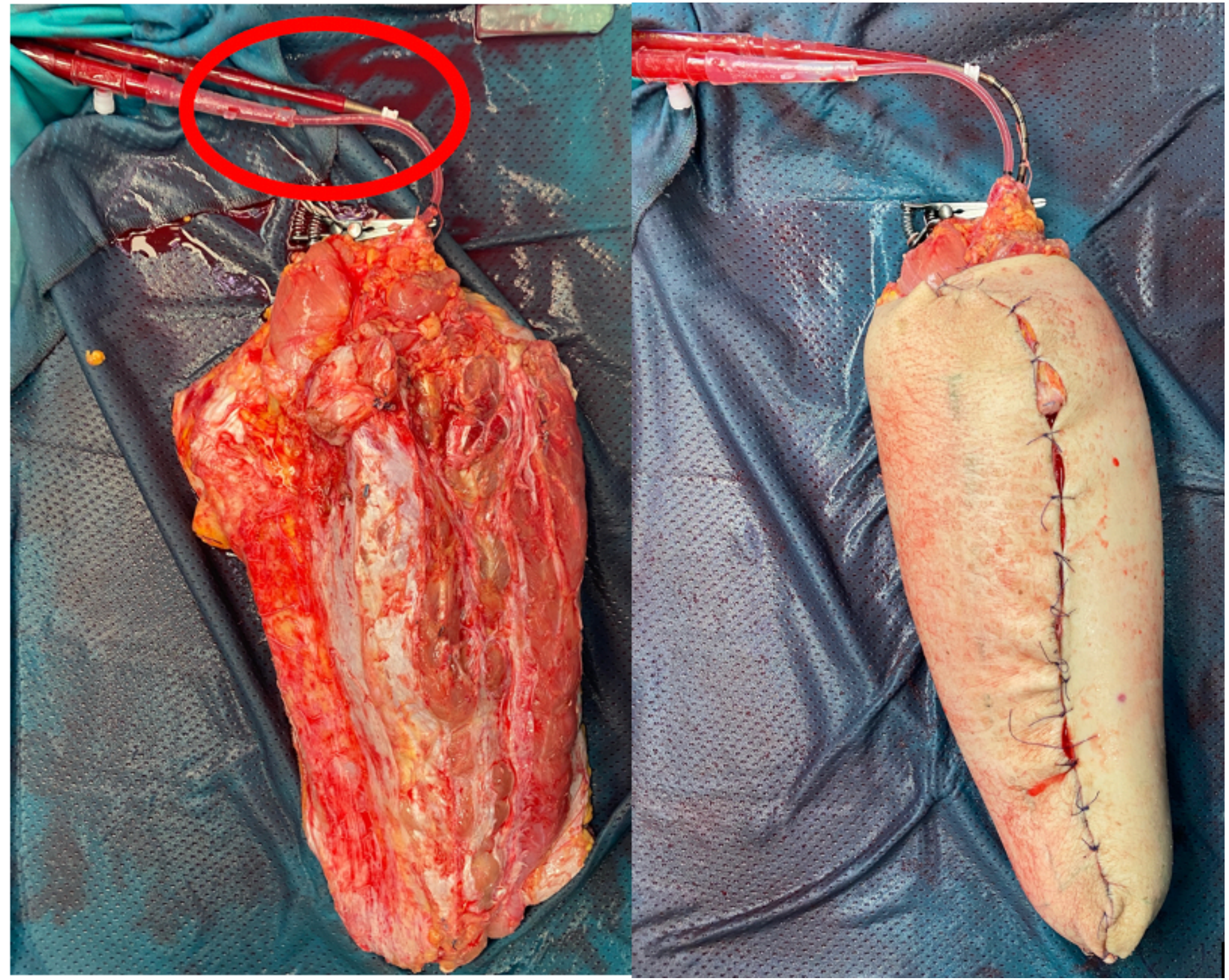


Figure 4

The flap was set aside on a separate surgical table and it was reperfused using the ECMO (ExtraCorporeal Membrane Oxygenation) system. Popliteal artery and vein were canulated (red circle), then the flap was closed in on itself and 2 units of blood red cells and saline solution were infused.

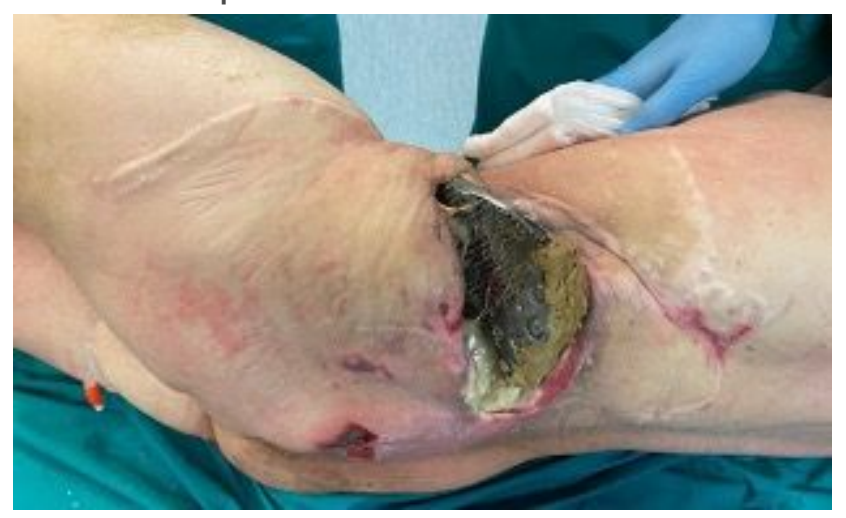

Figure 5

Postoperative rehabilitation. The patient was able to stand up and took steps with the help of the walker. 